\begin{tabular}{|c|c|}
\hline Title & Nanoscale mechanical contacts probed with ultrashort acoustic and thermal waves \\
\hline Author(s) & Dehoux, Thomas; Wright, Oliver B.; V oti, Roberto Li; Gusev, V italyi E. \\
\hline Citation & $\begin{array}{l}\text { Physical Review B, 80(23), } 235409 \\
\text { https://doi.org/10.1103/PhysRevB.80.235409 }\end{array}$ \\
\hline Issue Date & 2009-12-15 \\
\hline Doc URL & http:/hdl.handle.net/2115/42549 \\
\hline Rights & @2009 The A merican Physical Society \\
\hline Type & article \\
\hline File Information & PRB80-23_235409.pdf \\
\hline
\end{tabular}

Instructions for use 


\title{
gु \\ Nanoscale mechanical contacts probed with ultrashort acoustic and thermal waves
}

\author{
Thomas Dehoux and Oliver B. Wright* \\ Division of Applied Physics, Graduate School of Engineering, Hokkaido University, Sapporo 060-8628, Japan \\ Roberto Li Voti \\ Dipartimento di Energetica, Sapienza Università di Roma, Via A. Scarpa 16, Roma 00161, Italy \\ Vitalyi E. Gusev \\ Laboratoire de Physique de l'Etat Condensé, UMR CNRS 6087, Université du Maine, Le Mans F-72085, France
}

(Received 5 October 2009; published 8 December 2009)

\begin{abstract}
Using an ultrafast optical technique we measure coherent phonon-pulse reflection from-and heat flow across-a mechanical contact of nanoscale thickness between a thin metal film and a spherical dielectric indenter. Picosecond phonon wave packets at $\sim 50 \mathrm{GHz}$ returning from this interface probe the pressure distribution, the contact area, and the indentation profile to subnanometer resolution, revealing the film deformation in situ. These measurements and simultaneous thermal-wave imaging at $\gtrsim 1 \mathrm{MHz}$ are consistent with significant enhancement of phonon transport across the near-contact nanogap.
\end{abstract}

DOI: 10.1103/PhysRevB.80.235409

PACS number(s): 68.35.Ct, 78.20.Nv, 46.55.+d, 43.35.+d

\section{INTRODUCTION}

The properties of mechanical contacts between solids depend on how individual asperities mesh on the nanoscale, and a physical understanding is vital in engineering for the study of friction, lubrication, and electrical or heat conduction as well as in biology for the study of cell-membrane adhesion or joints. ${ }^{1,2}$ A generic approach to studying static mechanical contacts that facilitates the separation of elastic and plastic behaviors is the use of microscale or macroscale spherical (Hertz) indenters. ${ }^{1,3}$ This geometry is an apt choice for the lateral imaging of the contact by penetrating wave fields. $^{4}$

The contact area can be imaged by optical interferometry combined with microscopy. ${ }^{5}$ Ultrasonic imaging, applicable also to opaque materials, allows one to map in addition the interfacial stiffness or the contact pressure. ${ }^{6,7}$ However, with a spatial resolution on the order of the $\sim 0.1 \mathrm{~mm}$ acoustic wavelength at the typical maximum frequencies $\sim 30 \mathrm{MHz}$ used, one cannot effectively probe nanoscale deformations. Neither can one accurately study interfaces with nanoscale roughness. Likewise, probing mechanical contacts using modulated thermoreflectance up to the maximum frequencies $\sim 3 \mathrm{kHz}$ used is limited in spatial resolution by the $\sim 30 \mu \mathrm{m}$ thermal-diffusion length. ${ }^{8}$ One can image nanoindentation processes in a transmission electron microscope with subnanometer resolution, but this technique is not suited to spatially resolved mapping over the contact area. ${ }^{9}$

In this paper we implement much shorter acoustic and thermal wavelengths to map the deformation in or near a Hertz mechanical contact of nanoscale thickness. We introduce an in situ coherent phonon and thermal-profiling technique at frequencies $\sim 100 \mathrm{GHz}$ and $\gtrsim 1 \mathrm{MHz}$, respectively, more than 1000 times higher than previously used in contact mechanics.

\section{EXPERIMENTAL SETUP}

The setup is shown in Fig. 1(a). The sample is a 110-nm polycrystalline $\mathrm{Cr}$ film sputtered on a (0001) sapphire substrate of thickness $0.8 \mathrm{~mm}$. The sample lies on a rigid holder pierced with a $1.8-\mathrm{mm}$ radius hole for optical access. A sintered silicon-nitride sphere ${ }^{10}$ of diameter $D=5.96 \mathrm{~mm}$ is indented into the pristine $\mathrm{Cr}$ film using a high-precision displacement stage. The applied load, stable at each setting to $\pm 5 \%$ over several hours, is measured with a load cell. To investigate contacts with nanoscale separations, we use highly polished surfaces. The rms roughnesses of the film, indenter and substrate, determined by atomic force microscopy (AFM) with 40-nm step size [see Fig. 1(b)], are $\sigma_{f}=3 \mathrm{~nm}, \sigma_{i}=13 \mathrm{~nm}$, and $\sigma_{s}=1 \mathrm{~nm}$, respectively. ${ }^{11}$ Picosecond longitudinal coherent-phonon pulses are thermoelastically generated at the film-substrate $(f-s)$ interface with linearly polarized optical pump pulses of duration $200 \mathrm{fs}$, repetition frequency $80 \mathrm{MHz}$, wavelength $810 \mathrm{~nm}$ (optical reflectivity 0.23 ), and pulse energy $E=0.5 \mathrm{~nJ}$ from a Ti:sapphire mode-locked laser. ${ }^{12-14}$ This light is chopped at 1.1 $\mathrm{MHz}$ for thermal-wave excitation and lock-in detection. The reflectivity change $\delta R(t)$ is measured with frequencydoubled circularly polarized probe pulses of wavelength $\lambda_{p b}=405 \mathrm{~nm}$ (reflectivity $R_{0}=0.29$ ) and energy $0.06 \mathrm{~nJ}$ as a function of the pump-probe time delay $t$. The coaxial pump
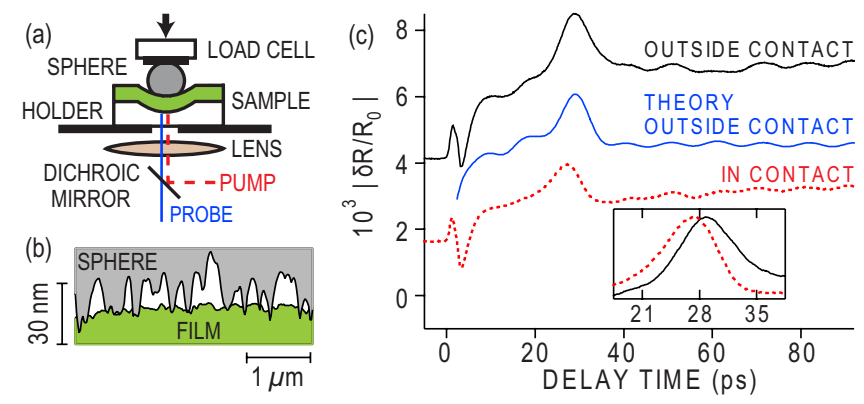

FIG. 1. (Color online) (a) Setup. (b) AFM traces. (c) $\left|\delta R(t) / R_{0}\right|$ at $F=4.2 \mathrm{~N}$. The theory out of contact is shown displaced. Inset: normalized experimental echoes. 
and probe beams are focused at the $f-s$ interface by a laterally scanned $\times 50$ objective lens to spots of radii $w_{1}=1.9 \mu \mathrm{m}$ and $w_{2}=1.1 \mu \mathrm{m}$ (intensity at $1 / e^{2}$ ), respectively [effective radius $w=\left(w_{1}^{2}+w_{2}^{2}\right)^{1 / 2}=2.2 \mu \mathrm{m}$ ]. Pertinent physical properties are the longitudinal sound velocity $v_{n}$, density $\rho_{n}$, Poisson's ratio $\nu_{n}$, Young's modulus $E_{n}$, thermal conductivity $\kappa_{n}$, and heat capacity $C_{n}$, with $n=i, f$, and $s$ for the indenter, film and substrate. ${ }^{15}$

\section{RESULTS FOR THE ACOUSTIC EXPERIMENTS}

\section{A. Shape of the acoustic echoes}

Figure 1(c) shows the amplitude $\left|\delta R(t) / R_{0}\right|$ (where $\delta R$ $<0)$ for the load $F=4.2 \mathrm{~N}$ in and out of contact. ${ }^{16} \delta R \propto E$ was checked up to pump-pulse energy $E=0.5 \mathrm{~nJ}$. For $t<0$, $|\delta R|$ is lower in contact due to thermal-wave effects, as discussed later. At $t=0$ we observe a peak followed by a dip and then a rise in $\left|\delta R / R_{0}\right|$ at $t=10-20$ ps. This is due to the cumulative effects of a transient-temperature rise ${ }^{17} \delta T$ $\sim 500 \mathrm{~K}(d R / d T<0)$ and strain generation. ${ }^{12,18}$

Initially a hydrostatic compressive stress field is generated in $\mathrm{Cr}$ over a depth $z_{a c} \sim 35 \mathrm{~nm}$ determined by the optical skin depth $\sim 30 \mathrm{~nm}$ (Ref. 19) and the electron diffusion depth $\sim 15 \mathrm{~nm} .{ }^{14}$ The acoustic impedances $Z_{n}=\rho_{n} v_{n}$ of $\mathrm{Cr}$ and $\mathrm{Al}_{2} \mathrm{O}_{3}$ imply a low film-substrate reflection coefficient $r_{f s}=\left(Z_{s}-Z_{f}\right) /\left(Z_{s}+Z_{f}\right)=-0.04$, so two unipolar compressive coherent-phonon pulses (of strain amplitude $\sim 10^{-3}$ ) are launched perpendicular to the $f-s$ interface in both materials. ${ }^{20}$ In $\mathrm{Al}_{2} \mathrm{O}_{3}$ these pulses create oscillations in $\delta R$ (visible after $40 \mathrm{ps}$ ) at the Brillouin frequency $2 n_{s} v_{s} / \lambda_{p b}$ $\approx 100 \mathrm{GHz}$, with $n_{s}=1.76$ the $\mathrm{Al}_{2} \mathrm{O}_{3}$ refractive index. ${ }^{20-22} \mathrm{In}$ $\mathrm{Cr}$ a broadband picosecond strain pulse of frequency $0<f$ $\lesssim 100 \mathrm{GHz}$ (Ref. 23) and wavelength $\Lambda \gtrsim 70 \mathrm{~nm}$ is reflected from the film-indenter $(f-i)$ interface with a reflection coefficient $r_{f i}$ (determined later). This pulse is detected at the $f$-s interface as an echo at $\sim 30$ ps. Because of the low value of $r_{f s}$, only one echo is resolved. Echoes normalized using $r_{f i}$ in and out of contact are shown in Fig. 1(c). When in contact the echo arrives earlier, is reduced in height, and is asymmetrically distorted, although the echo spectrum is not significantly changed. The echo shape depends on the longitudinal strain $\eta$ that in the near-surface region can be written in the approximate form ${ }^{12,14}$

$$
\eta(z, t) \propto \theta\left(z-v_{f} t\right) \exp \left[\left(v_{f} t-z\right) / z_{a c}\right]
$$

where $\theta$ is the unit step function and $z$ is the depth. We combine this equation with an ultrasonic attenuation that rises quadratically with the frequency for $\mathrm{Cr}$ in the $\mathrm{GHz}$ range. ${ }^{14}$ The resulting $\eta(z, t)$ allows one to derive the expected reflectivity variation that arises through the photoelastic effect. ${ }^{12,24}$ The central curve in Fig. 1(c) shows the predicted $\left|\delta R(t) / R_{0}\right|$ out of contact using the photoelastic constant $p_{12}^{s}=-0.03$ for $\mathrm{Al}_{2} \mathrm{O}_{3}$ (Ref. 21) and the fit $p_{12}^{f}$ $=0.28+0.00 \mathrm{i}$ for $\mathrm{Cr}$. For the $\mathrm{Cr}$ film in this study, ultrasonic attenuation $\beta=600 f^{2} \mathrm{~m}^{-1}$ ( $f$ in $\mathrm{GHz}$ ) gives the best fit to the echo with a film thickness $h=110 \mathrm{~nm} .^{14,25}$
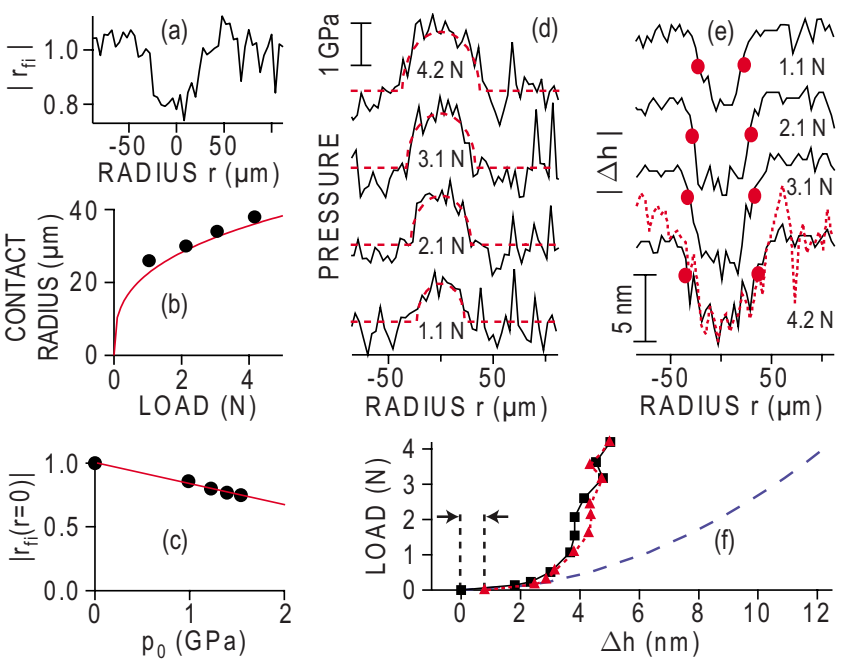

FIG. 2. (Color online) (a) Acoustic reflection coefficient $\left|r_{f i}\right|$ vs $r$ at $F=2.1$ N. (b) Contact radius from $\left|r_{f i}\right|$ (dots) and Hertz theory for $\mathrm{Al}_{2} \mathrm{O}_{3}-\mathrm{Si}_{3} \mathrm{~N}_{4}$ (line). (c) $\left|r_{f i}(r=0)\right|$ against $p_{0}$ (dots) with fit (line). (d) Pressure $p$ vs $r$ (solid lines) and Hertz theory (dashed lines). (e) Film indentation $\Delta h$. Dots: Hertz contact radii. Dashed line: $-p(r)$ for $F=4.2$ N. (f) $F$ vs $|\Delta h|$ at $r=0$ for loading ( $\square$ ) and unloading (A) compared with theory (dashes). Arrows: permanent deformation.

\section{B. Profiles of the acoustic reflection coefficient}

A left-right average of the base of the echo determines the echo height. Setting $r_{f i}=-1$ outside the contact determines the in-contact value. We plot $\left|r_{f i}\right|$ vs radial coordinate $r$ in Fig. 2(a) for load $F=2.1 \mathrm{~N}$ in $4-\mu \mathrm{m}$ steps. The fluctuations, showing some correlation with scans at different $F$, are caused by surface roughness. Over the contact, $\left|r_{f i}\right|$ decreases to 0.8 . For a perfect contact, $r_{f i}=r_{f i}^{\text {th }}=\left(Z_{i}-Z_{f}\right) /\left(Z_{i}+Z_{f}\right)=$ -0.17 . Interstitial air gaps, shown in Fig. 1(b), result in a larger $\left|r_{f i}\right|$. As $F$ increases, the total asperity (i.e., real) contact area increases, and $\left|r_{f i}(r=0)\right|$ decreases. We can empirically fit our data with $\left|r_{f i}(r=0)\right|=1-(F / 110)^{0.4}$, with $F$ in $\mathrm{N}$. To obtain further insight into the local variations within the contact, we must calibrate our results in terms of pressure.

We first estimate the contact radius $a$ from Hertz theory ${ }^{1}$ for a sphere indenting a frictionless half space

$$
a^{3}=3 F D / 8 E^{*},
$$

where $E^{*}=\left[\left(1-\nu_{i}^{2}\right) / E_{i}+\left(1-\nu_{s}^{2}\right) / E_{s}\right]^{-1}=200 \mathrm{GPa}$ is the effective modulus. We derive $a$ from $\left|r_{f i}(r)\right|$ and compare it to theory in Fig. 2(b). The data lie above the predictions, consistent with theories for elastically indented thin films for $E_{f} / E_{s}<1 .{ }^{26}$ To apply Hertz theory, the combined surface roughness $\sigma=\left(\sigma_{1}^{2}+\sigma_{2}^{2}\right)^{1 / 2} \approx 13 \mathrm{~nm}$ should also be considered. In our case the roughness parameter $\alpha=\sigma D / 2 a^{2}$ $<0.07 \ll 1$ for $F>1.1 \mathrm{~N}$, so Hertz theory is valid. ${ }^{1}$ We thus use the Hertz pressure

$$
p(r)=p_{0}\left[1-(r / a)^{2}\right]^{1 / 2},
$$

with maximum value $p_{0}=3 F / 2 \pi a^{2}$, to derive $\left|r_{f i}\left(p_{0}\right)\right|$ from the measured $\left|r_{f i}(F)\right|$ at $r=0$. We see a linear decrease $\left|r_{f i}\left(p_{0}\right)\right| \approx 1-p_{0} / p_{1}$ in Fig. 2(c) with $p_{1}=6.1 \mathrm{GPa}$. This pro- 
vides a calibration to obtain $p(r)=p_{1}\left[1-\left|r_{f i}(r)\right|\right]$, showing good agreement with theory in Fig. 2(d). Before discussing these results in detail we first present the results for film indentation.

\section{Profiles of the indentation}

Uniquely, with picosecond ultrasonics we can directly extract the film indentation $\Delta h=v_{f} \Delta \tau / 2$ from the echo arrivaltime variation $\Delta \tau$ during loading. ${ }^{27} \Delta h(r)$, plotted in Fig. 2(e), varies over regions slightly larger than the contact radii $a$, as expected. Striking correlation at each load $F$ between the fluctuations in $\Delta h(r)$ and $-p(r)$ [see dashed line in Fig. $2(\mathrm{e})]$ confirms that these are indeed induced by roughness. Finer scale data at $r=0$ is shown in Fig. 2(f) for a first loadunload cycle in steps of $0.5 \mathrm{~N}$ lasting $10 \mathrm{~min}$ per point. The overall shape of $F$ vs $|\Delta h|$ is evidently governed by elasticity. The application of linear elasticity theory for a thin $\mathrm{Cr}$ film on a rigid substrate indented by a rigid sphere leads to the following equation: ${ }^{28}$

$$
F=\pi D \rho_{f} v_{f}^{2} \Delta h^{2} / 2 h .
$$

The predicted load-indentation curve is shown by the dashed line in Fig. 2(f). Unsurprisingly, the measured $|\Delta h|$ $(<6 \mathrm{~nm})$ is smaller because of the indenter and substrate elasticity. In comparison, the deformation of the substrateindenter system is much larger: $\delta=2 a^{2} / D>180 \mathrm{~nm}$ for $F>1.1$ N. $^{1}$

A permanent plastic deformation $\sim 1 \mathrm{~nm}$ remains after unloading [see arrows in Fig. 2(f)]. However, the Vickers hardness $H \approx 15 \mathrm{GPa}$ of thin-film $\mathrm{Cr},{ }^{29}$ the softer material, indicates that the maximum pressure $p_{0}=1.5 \mathrm{GPa}$ is not sufficient for bulk-film yielding. ${ }^{1}$ This justifies the use of linear elastic theory for the bulk deformation of $\mathrm{Cr}$. In order to understand the origin of the plasticity, one should consider the asperity deformation. For our Gaussian distribution of asperity heights, this is governed by the so-called plasticity index: $:^{2,30}$

$$
\psi=\left(E^{*} / H\right)\left(\sigma / r_{a}\right)^{1 / 2} .
$$

Here $\psi \gtrsim 3$, where $r_{a} \lesssim 0.3 \mu \mathrm{m}$ is the AFM-measured average $\mathrm{Si}_{3} \mathrm{~N}_{4}$ asperity radius; $\psi>1$ indicates $\mathrm{Cr}$ plastic compression by the harder $\mathrm{Si}_{3} \mathrm{~N}_{4}$. For noninteracting asperities, overall force balance dictates that $S_{r} H=S_{0} p$, where $S_{r}$ and $S_{0}$ are the real and nominal contact areas, respectively. ${ }^{2,4}$ At the maximum load $F=4.2 \mathrm{~N}, S_{r} / S_{0} \approx 0.1$ at $r=0$. Using the measured $\mathrm{Si}_{3} \mathrm{~N}_{4}$ rms roughness $\sigma_{i}=13 \mathrm{~nm}$ and rms surface slope $0.2 \mathrm{rad}$, we estimate a corresponding contact clearance $u \sim 15 \mathrm{~nm}$, with an average asperity contact radius $R_{a}$ $\sim 60 \mathrm{~nm}$ at a density $N_{a} \sim 10 \mu \mathrm{m}^{-2}$. ${ }^{31}$ This order of magnitude for $u$ demonstrates that picosecond ultrasonics is exquisitely sensitive to the closing of nanoscale gaps between solids.

\section{Interpretation of the results for acoustic reflection coefficient}

Precise modeling of $\left|r_{f i}\right|$ from contacts is complicated by the broadband strain pulse. At very low frequencies, it is common to assume the presence of a thin interlayer of thickness $l$, density $\rho$, and longitudinal sound velocity $v$, or a contact stiffness per unit area $K=\rho v^{2} / l .^{7,32-35}$ For plastic deformation of asperities, each of Hertz stiffness $2 E^{*} R_{a}$, ${ }^{1,6}$ $K=2 E^{*} R_{a} N_{a} \leq 0.2 \mathrm{GPa} \mathrm{nm}^{-1}$. This implies a reduced $\left|r_{f i}\right|$ for $f \lesssim K /\left(2 \pi Z_{f}\right)=0.7 \mathrm{GHz}(\Lambda \gtrsim 10 \mu \mathrm{m})$. However, this results in a negligible change in $\left|r_{f i}\right|$, inconsistent with the observed $\sim 25 \%$ reduction at $F=4.2 \mathrm{~N}$. Since wavelengths $\Lambda \gtrsim 70 \mathrm{~nm} \sim R_{a}$ are present at the $f-i$ interface, this model should be replaced by a theory of acoustic scattering from nanoscale contacts.

To this end, consider the diffraction of a strain pulse, of radial dependence $\eta_{1} \propto \exp \left(-r^{2} / w_{1}^{2}\right)$ governed by the pump spot of radius $w_{1}$, generated at the $f-s$ interface. The $z$ and $t$ dependences in $\eta_{1}$ are implicit here. This pulse reaches the $f-i$ interface in the near field without lateral spreading since the $\mathrm{Cr}$ thickness satisfies the relation $h \ll w_{1}^{2} / \Lambda$. Provided $R_{a} \gg \Lambda / 2 \pi$, reasonable only at frequencies $f \gtrsim 20 \mathrm{GHz}$, each contacting asperity can be approximated to have a reflection coefficient $\left|r_{f i}^{\mathrm{th}}\right|=0.17$, assuming negligible mode conversion. ${ }^{36}$ We define a corresponding normalized top-hat function $T\left(\mathbf{r}-\mathbf{d}_{i}\right)$, where $\mathbf{r}$ and $\mathbf{d}_{i}$ are, respectively, the inplane position vector and that of the $i$ th asperity. The reflected field $\eta_{2}(\mathbf{r})$ at the $f-i$ interface is then

$$
\eta_{2}(\mathbf{r})=\eta_{1}(\mathbf{r})-\left(1-\left|r_{f i}^{\mathrm{th}}\right|\right) \sum_{i} T\left(\mathbf{r}-\mathbf{d}_{i}\right) \eta_{1}\left(\mathbf{d}_{i}\right) .
$$

Applying Babinet's principle to the diffraction of this discontinuous source-analogous to a holey cheese-over the propagation length $h$ and positing randomly distributed asperities over the probe spot of radius $w_{2}$, we get the following simple result based on relative areas:

$$
\left|r_{f i}\right|=1-\left(1-\left|r_{f i}^{\mathrm{th}}\right|\right) \pi R_{a}^{2} N_{a},
$$

where $h \ll w_{2}^{2} / \Lambda$. Using $\pi R_{a}^{2} N_{a}=S_{r} / S_{0}$, this implies

$$
\left|r_{f i}(r=0)\right|=1-\left(1-\left|r_{f i}^{\mathrm{th}}\right|\right) p_{0} / H .
$$

However, even using extrapolation to $f=0$ only leads to an $8 \%$ drop in $\left|r_{f i}\right|$ at $F=4.2 \mathrm{~N}$. So this model of acoustic diffraction only partially explains our finding of a $\sim 25 \%$ reduction in $\left|r_{f i}\right|$ at this load. An additional mechanism for enhanced acoustic transmission or scattering should therefore be sought.

The above model is limited to high frequencies but also in that it ignores the asperity vibrational eigenmodes. ${ }^{37,38}$ Even for frequencies well below their longitudinal resonance $\sim v_{i} /(2 u) \sim 300 \mathrm{GHz}$, enhanced transmission and echo distortion can result when the lateral mode structure and the contribution of evanescent waves to $r_{f i}$ are considered. The required numerical analysis of this process is beyond the scope of this paper. It is clear, however, that our experimental approach is apt for probing the physics of acoustic diffraction from and resonance in nanoasperities.

\section{RESULTS FOR THE THERMAL EXPERIMENTS}

\section{A. Thermal profiles of the contact}

To obtain contact profiles based mainly on thermal waves with diffusion lengths $\mu_{n}=\left(2 \kappa_{n} / C_{n} \omega\right)^{1 / 2} \lesssim 3 \mu \mathrm{m}$, we make 


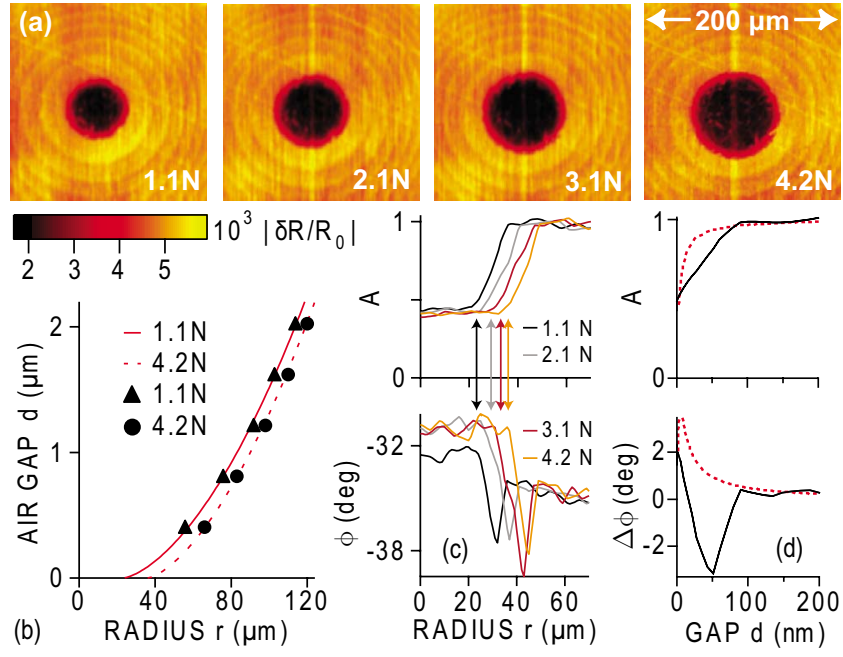

FIG. 3. (Color online) (a) $\left|\delta R / R_{0}\right|$ images of the contact. (b) Measured air gap $(\boldsymbol{\Delta}, \boldsymbol{O})$ and Hertz theory (lines) for two loads. (c) Normalized amplitude and phase for $t=-10$ ps. Arrows: contact radii. (d) Normalized amplitude and phase difference for $1.1 \mathrm{~N}$ and 3D thermal-diffusion theory (dashed lines).

radial scans of $\left|\delta R / R_{0}\right|$ at $t=-10$ ps to avoid acoustic effects. The probed temperature variation is $A \sin (\omega t+\phi)$, where $\sin \omega t$ is in phase with the chopping at $1.1 \mathrm{MHz}$ and $\phi$ is the phase lead recorded by the lock-in amplifier. $\left|\delta R / R_{0}\right| \propto A$ [ $\sim 70 \mathrm{~K}$ (Ref. 17)], normalized with respect to its out-ofcontact value, and phase lead $\phi$ are plotted vs $r$ in Fig. 3(c). The contact radius, determined from the initial rise in $A$ or decrease in $\phi$ [arrows in Fig. 3(c)], agrees with Hertz theory. At larger $r, A$ saturates, whereas $\phi$ shows a sharp minimum. Both remain constant over the contact area for $F \geq 1.1 \mathrm{~N}$.

Confirmation of these results in two spatial dimensions is shown for $\left|\delta R / R_{0}\right|$ in Fig. 3(a) for a $200 \times 200 \mu \mathrm{m}^{2}$ region scanned in $1-\mu \mathrm{m}$ steps. ${ }^{39} \mathrm{We}$ resolve the circular contact area as well as low-contrast Newton's rings. ${ }^{5}$ These arise from variations in pump-light absorption on residual transmission to the $f-i$ air gap. ${ }^{19}$ The derived air gap agrees with Hertz theory [see Fig. 3(b)].

\section{B. Interpretation of the thermal profiles}

The three-dimensional (3D) thermal-diffusion equation was solved for a sinusoidal spatially Gaussian heat source (of radius $w=2.2 \mu \mathrm{m})$ at the $f$-s interface with an air gap $d$ below a flat and parallel (in practice to $<1^{\circ}$ ) $\mathrm{Si}_{3} \mathrm{~N}_{4}$ surface. The effect of the periodic laser-pulse trains when probing at the chopping frequency was also included using a sum arising from harmonics of the $80 \mathrm{MHz}$ repetition rate. ${ }^{40}$ Harmonics up to order $\sim 2000$ (corresponding to $\sim 160 \mathrm{GHz}$ thermal waves) give a non-negligible contribution here, but only those with frequencies $\lesssim 1 \mathrm{GHz}\left(\mu_{n} \geq h\right)$ probe the $f$-i interface.

The predictions for $A(d)$ and $\phi(d)$ are compared to experiment on a normalized scale in Fig. 3(d), assuming literature values of thermal properties. ${ }^{15,41}$ Thermal-boundary resistances $R_{f s}=1.3 \times 10^{-7}$ and $R_{f i}=1.0 \times 10^{-7} \mathrm{~K} \mathrm{~m}^{2} \mathrm{~W}^{-1}$ at the $f$ - $s$ and $f-i$ interfaces (the latter for $d=0$ only) are determined by matching the contrast in $A$ and the difference $\Delta \phi$ in $\phi$ in and out of contact. ${ }^{42}$ Standard models ${ }^{43}$ based on acoustic mismatch, constriction, and electron-phonon resistances give values of a similar order. However, in experiment $\phi(d)$ shows a minimum. ${ }^{44} A(d)$ also varies more slowly: ${ }^{45}$ this points to enhanced heat transport through the gap for $d$ $\$ 50 \mathrm{~nm}$. At $293 \mathrm{~K}$ and $1 \mathrm{~atm}$, the molecular mean-free path and the Knudsen number are $L=60 \mathrm{~nm}$ and $\mathrm{Kn}=L / d>0.6$ (for $d<100 \mathrm{~nm}$ ). ${ }^{46}$ For this "near-continuum" regime, ballistic heat transport competes strongly with diffusion. The ratio of the thermal conductances in the diffusive $\left(K_{d}=\kappa_{a} / d\right)\left(\right.$ Ref. 41) and near-continuum $\left(K_{n c}\right)$ regimes is

$$
\frac{K_{d}}{K_{n c}}=1+\frac{4 \gamma}{\gamma+1} \frac{\alpha_{f}+\alpha_{i}-\alpha_{f} \alpha_{i}}{\alpha_{f} \alpha_{i}} \frac{\mathrm{Kn}}{\operatorname{Pr}},
$$

with $\gamma=7 / 5$ for a diatomic gas and $\operatorname{Pr}=0.72$ the Prandtl number in air. ${ }^{2,46} \alpha_{f}$ and $\alpha_{i}$ are the film and indenter accommodation coefficients $(<1)$. Gap heat transport should therefore be overestimated $\left(K_{d} / K_{n c} \geqslant 3\right)$ (Ref. 47), so kinetic theory fails to explain the anomaly in $A$. Another possibility is near-field thermal radiation. ${ }^{48}$ But to account for our results would require a $K_{d} / 4 \sigma_{B} T^{3} \sim 10^{5}$ enhancement compared to the blackbody value, where $\sigma_{B}$ is the StefanBoltzmann constant, an implausible factor for dissimilar materials. Although Hertz theory explains the acoustically determined contact radius $a$, it may be that surface roughness plays a role in explaining the anomalies. The roughness parameter $\alpha \sim 0.05$ implies some contact even at radii $1.3 a{ }^{1}$ Compared to the coherent acoustic phonons, the efficient transmission of the much shorter wavelength incoherent thermal phonons through the asperities should therefore broaden the thermal-wave-probed contact area. However, the anomalies in amplitude and phase occur at a gap thickness of $\sim 50 \mathrm{~nm}$, a value that is seemingly too large to be accounted for by the smaller rms surface roughnesses of the contacting $\mathrm{Cr}$ and $\mathrm{Si}_{3} \mathrm{~N}_{4}$. A theory accounting for the detailed geometry of the asperity penetration into the gap, an intermediate region in which asperities and air coexist, is required. To elucidate the thermal anomalies we observe, measurements with different indenters in evacuated chambers should be carried out to reveal the physics of asperity deformation and thermal transport through the nanowire-like connections that connect the contacting bodies.

\section{CONCLUSION}

In conclusion, we demonstrate that nanoscale acoustic waves excited with ultrashort optical pulses show enhanced coherent phonon transport between or enhanced scattering from solids in mechanical contact. In addition, we demonstrate that optically excited microscale thermal waves show enhanced incoherent phonon transport across the gap between solids under near-contact conditions. These findings should be investigated in the future by experiments on different contacting materials, on a range of surface roughnesses, and with a variety of gases or a vacuum in the contact region. In addition, further measurements using coated 
transparent indenters with the pump and probe light incident on opposite sides of the interface will allow phonon transmission to be monitored. Accessing nanometer indentation with high-frequency phonons also opens the way to mapping deformation or plastic flow in a wide variety of materialswhether metallic, semiconductor, or dielectric — and to direct probing of the effects of wear and friction in nanomechanical systems. *assp@kino-ap.eng.hokudai.ac.jp

${ }^{1}$ K. L. Johnson, Contact Mechanics (Cambridge University Press, Cambridge, 1985).

${ }^{2}$ C. V. Madhusudana, Thermal Contact Conductance (Springer, New York, 1996).

${ }^{3}$ M. V. Swain, Mater. Sci. Eng., A 253, 160 (1998).

${ }^{4}$ P. W. O'Callaghan and S. D. Probert, Wear 29, 120 (1987).

${ }^{5}$ A. Ovcharenko, G. Halperin, G. Verberne, and I. Etsion, Tribol. Lett. 25, 153 (2007).

${ }^{6}$ K. Kendall and D. Tabor, Proc. R. Soc. London, Ser. A 323, 321 (1971).

${ }^{7}$ B. W. Drinkwater, R. S. Dwyer-Joyce, and P. Cawley, Proc. R. Soc. London, Ser. A 452, 2613 (1996).

${ }^{8}$ Y. Ohsone, G. Wu, J. Dryden, F. Zok, and A. Majumdar, ASME J. Heat Transfer 121, 954 (1999).

${ }^{9}$ M. S. Bobji, J. B. Pethica, and B. J. Inkson, J. Mater. Res. 20, 2726 (2005)

${ }^{10}$ From Toshiba Materials Co. datasheet (2008).

${ }^{11}$ The respective rms surface slopes are $4^{\circ}, 11^{\circ}$, and $1^{\circ}$.

${ }^{12}$ C. Thomsen, H. T. Grahn, H. J. Maris, and J. Tauc, Phys. Rev. B 34, 4129 (1986).

${ }^{13}$ O. B. Wright and K. Kawashima, Phys. Rev. Lett. 69, 1668 (1992).

${ }^{14}$ T. Saito, O. Matsuda, and O. B. Wright, Phys. Rev. B 67, 205421 (2003).

${ }^{15} \rho_{i}=3.2, \rho_{f}=7.2$, and $\rho_{s}=4.0 \mathrm{~g} \mathrm{~cm}^{-3} ; \nu_{i}=0.29, \nu_{f}=0.21$, and $\nu_{s}$ $=0.23 ; E_{i}=320, E_{f}=280$, and $E_{s}=440 \mathrm{GPa} ; v_{i}=10.6, v_{f}=6.62$, and $v_{s}=11.1 \mathrm{~km} \mathrm{~s}^{-1} ; \kappa_{i}=28, \kappa_{f}=14$, and $\kappa_{s}=45 \mathrm{~W} \mathrm{~m}^{-1} \mathrm{~K}^{-1}$; $C_{i}=2.1 \times 10^{6}, \quad C_{f}=3.2 \times 10^{6}, \quad$ and $C_{s}=3.4 \times 10^{6} \mathrm{~J} \mathrm{~m}^{-3} \mathrm{~K}^{-1}$ (taken at $300 \mathrm{~K}$ ). $\kappa_{f}$ is $1 / 7$ of the single-crystal value. See Refs. $10,14,22,49$, and 50 .

${ }^{16}$ In the contact region, $R_{0}$ increases by $\sim 5 \%$.

${ }^{17}$ S. Kashiwada, O. Matsuda, J. J. Baumberg, R. Li Voti, and O. B. Wright, J. Appl. Phys. 100, 073506 (2006).

${ }^{18}$ O. B. Wright and T. Hyoguchi, Opt. Lett. 16, 1529 (1991).

${ }^{19}$ Refractive indices of $\mathrm{Cr} 2.6+2.6 i$ at $405 \mathrm{~nm}$ and $3.8+2.0 i$ at 810 $\mathrm{nm}$ were measured by ellipsometry.

${ }^{20}$ O. B. Wright, Opt. Lett. 20, 632 (1995).

${ }^{21}$ D. Lide, CRC Handbook of Chemistry and Physics (CRC, Boca Raton, 1999).

${ }^{22}$ O. L. Anderson, in Physical Acoustics, edited by W. P. Mason (Academic, New York, 1965), Vol. 3B, pp. 43-95.

${ }^{23}$ The amplitude decreases by $1 / e^{2}$ over this range of $f$.

${ }^{24}$ O. Matsuda and O. B. Wright, J. Opt. Soc. Am. B 19, 3028 (2002).

${ }^{25}$ Echo smoothing $\propto \exp \left[-2\left(\pi f \delta h / v_{f}\right)^{2}\right]$ by reflection from $\mathrm{nm} \mathrm{rms}$ variations $\delta h$ in $h$ is negligible. See S. A. Akhmanov and V. E. Gusev, Sov. Phys. Usp. 35, 153 (1992).

${ }^{26}$ H. Y. Yu, S. C. Sanday, and B. B. Rath, J. Mech. Phys. Solids 38, 745 (1990).

${ }^{27}$ The variation in $v_{f}$ with pressure is negligible here.
${ }^{28}$ M. J. Jaffar, Proc. Inst. Mech. Eng., Part C: J. Mech. Eng. Sci. 211, 549 (1997).

${ }^{29}$ B. Jönsson and S. Hogmark, Thin Solid Films 114, 257 (1984).

${ }^{30}$ J. A. Greenwood and J. B. P. Williamson, Proc. R. Soc. London, Ser. A 295, 300 (1966).

${ }^{31}$ Y. Kimura, Wear 15, 47 (1970).

${ }^{32}$ J.-Y. Kim, A. Baltazar, and S. I. Rokhlin, J. Mech. Phys. Solids 52, 1911 (2004).

${ }^{33}$ A. I. Lavrentyev and S. I. Rokhlin, J. Acoust. Soc. Am. 103, 657 (1998).

${ }^{34}$ J. M. Baik and R. B. Thompson, J. Nondestruct. Eval. 4, 177 (1984).

${ }^{35}$ Such theories have also been applied in picosecond ultrasonics to ultrathin layers. See, Ref. 13 or G. Tas, J. J. Loomis, H. J. Maris, A. A. Bailes, and L. E. Seiberling, Appl. Phys. Lett. 72, 2235 (1998)

${ }^{36}$ Conversion to shear is negligible. See, e.g., Y. C. Angel and J. D. Achenbach, ASME J. Appl. Mech. 52, 33 (1985).

${ }^{37}$ J. Christensen, L. Martin-Moreno, and F. J. Garcia-Vidal, Phys. Rev. Lett. 101, 014301 (2008).

${ }^{38}$ Acoustic transmission at $\sim 50 \mathrm{GHz}$ only occurs for air gaps $<0.1$ pm (see Ref. 7). Intermittent contact at our acoustic displacements $\sim 0.1 \mathrm{~nm}$ has negligible influence as does a $\sim 2 \mathrm{~nm}$ water layer under our conditions of $35 \%$ humidity. See J. Israelachvili, Intermolecular and Surface Forces (Academic, London, 1985).

${ }^{39}$ This data was taken at $t=40 \mathrm{ps}$. The contrast at this time is mainly determined by the temperature changes.

${ }^{40}$ See A. J. Schmidt, X. Chen, and G. Chen, Rev. Sci. Instrum. 79, 114902 (2008). Harmonics contribute $<40 \%$ to normalized $A$ and a shift of $<4^{\circ}$ in $\phi$.

${ }^{41}$ For air at $300 \mathrm{~K}, \rho_{a}=1.01 \mathrm{~kg} \mathrm{~m}^{-3}, \kappa_{a}=0.026 \mathrm{~W} \mathrm{~m}^{-1} \mathrm{~K}^{-1}$, and $C_{a}=1.02 \times 10^{3} \mathrm{~J} \mathrm{~m}^{-3} \mathrm{~K}^{-1}$ (see Ref. 21).

${ }^{42}$ Reduction in $R_{f i}$ leads to similar results, consistent with the observed saturation in $A$ and $\phi$ for $F \geq 1.1 \mathrm{~N}$.

${ }^{43}$ R. S. Prasher and P. E. Phelan, J. Appl. Phys. 100, 063538 (2006).

${ }^{44}$ The predicted $\phi$ in and out of contact are $-14.4^{\circ}$ and $-17.9^{\circ}$.

${ }^{45}$ Preliminary measurements of $A(d)$ for an $h=215 \mathrm{~nm}$ and $\sigma_{f}$ $=7 \mathrm{~nm}$ film [T. Dehoux, R. Li Voti, and O. B. Wright (unpublished)] showed similar trends.

${ }^{46}$ E. H. Kennard, Kinetic Theory of Gases (McGraw-Hill, New York, 1938).

${ }^{47}$ P. M. Patel, D. P. Almond, and H. Reiter, Appl. Phys. B: Lasers Opt. 43, 9 (1987).

${ }^{48}$ J.-P. Mulet, K. J. Joulain, R. Carminati, and J.-J. Greffet, Microscale Thermophys. Eng. 6, 209 (2002).

${ }^{49}$ W. C. Oliver and G. M. Pharr, J. Mater. Res. 7, 1564 (1992).

${ }^{50}$ M. Tomoda, O. B. Wright, and R. Li Voti, Appl. Phys. Lett. 91, 071911 (2007). 